Max-Planck-Institut für demografische Forschung

Max Planck Institute for Demographic Research

Konrad-Zuse-Strasse 1 - D-18057 Rostock · GERMANY

Tel +49 (0) 3812081 - 0; Fax +49 (0) 3812081 - 202;

http://www.demogr.mpg.de

MPIDR WORKING PAPER WP 2006-023

AUGUST 2006

\title{
On asymptotic failure rates in bivariate frailty competing risks models
}

Maxim Finkelstein (FinkelM@sci.uovs.ac.za)

Veronica Esaulova

This working paper has been approved for release by: James W. Vaupel (jwv@ demogr.mpg.de)

Head of the Laboratory of Survival and Longevity.

(C) Copyright is held by the authors.

Working papers of the Max Planck Institute for Demographic Research receive only limited review. Views or opinions expressed in working papers are attributable to the authors and do not necessarily reflect those of the Institute. 


\title{
On Asymptotic Failure Rates in Bivariate Frailty Competing Risks Models
}

\author{
Maxim Finkelstein \\ Department of Mathematical Statistics, University of the Free State \\ PO Box 339, 9300 Bloemfontein, Republic of South Africa, \\ (email: FinkelM.SCI@mail.uovs.ac.za), \\ Max Planck Institute for Demographic Research, Rostock, Germany \\ Veronica Esaulova \\ Otto von Guericke University, Magdeburg
}

\begin{abstract}
A bivariate competing risks problem is considered for a rather general class of survival models. The lifetime distribution of each component is indexed by a frailty parameter. Under the assumption of conditional independence of components the correlated frailty model is considered. The explicit asymptotic formula for the mixture failure rate of a system is derived. It is proved that asymptotically, as $t \rightarrow \infty$, the remaining lifetimes of components tend to be independent in the defined sense. Some simple examples are discussed.
\end{abstract}

Keywords: correlated frailty, mixture failure rate, competing risks, bivariate distributions

\section{Introduction}

It is well known that mixtures of distributions is a convenient tool for analyzing univariate frailty models. As monotonicity properties of the mixture failure rate can differ dramatically from those of the baseline failure rate, 
this topic was thoroughly investigated in the literature (see Block et al, 1993, Lynch, 1999, Finkelstein and Esaulova, 2001, Badia et al, 2001 to name a few). Special attention was also paid to asymptotic behavior of mixture failure rates (Block et al, 2003, Finkelstein and Esaulova, 2006, Shaked and Spizzichino, 2001).

In our paper (Finkelstein and Esaulova, 2006) a general class of lifetime models with frailties was considered. The basic model for $F(t, z)$ - the absolutely continuous cumulative distribution function (Cdf) of a lifetime random variable $T$, was defined as

$$
\Lambda(t, z)=A(z \phi(t))+\psi(t)
$$

where $\Lambda(t, z)=\int_{0}^{t} \lambda(u, z) d u$ is the corresponding cumulative failure rate and $z$ is a realization of fraily $Z$. The general assumptions on the functions involved were rather natural: $A(s), \phi(t)$ and $\psi(t)$ are differentiable, the right hand side of (1) is non-decreasing in $t$ and increases to infinity as $t \rightarrow \infty$, and that $A(z \phi(0))+\psi(0)=0$.

The widely used in reliability, survival analysis and risk analysis proportional hazards $(\mathrm{PH})$, additive hazards $(\mathrm{AH})$ and accelerated life (ALM) models, are the obvious specific cases of (1):

\section{PH (multiplicative) Model:}

Let

$$
A(u) \equiv u, \quad \phi(t)=\Lambda(t), \quad \psi(t)=0 .
$$

Then

$$
\lambda(t, z)=z \lambda(t), \quad \Lambda(t, z)=z \Lambda(t) .
$$

\section{Accelerated Life Model:}

Let

$$
A(u) \equiv \Lambda(u), \quad \phi(t)=t, \quad \psi(t)=0 .
$$

Then

$$
\Lambda(t, z)=\int_{0}^{t z} \lambda(u) d u=\Lambda(t z), \quad \lambda(t, z)=z \lambda(t z) .
$$

\section{AH Model:}

Let

$$
A(u) \equiv u, \quad \phi(t)=t, \quad \psi(t) \text { is increasing, } \quad \psi(0)=0 .
$$


Then

$$
\lambda(t, z)=z+\psi^{\prime}(t), \quad \Lambda(t, z)=z t+\psi(t) .
$$

Under the stated assumptions and using some additional technical conditions for the pdf of the frailty $Z$, we derived exact asymptotic relations for the corresponding mixture failure rate $\lambda_{m}(t)$ as $t \rightarrow \infty$ (Finkelstein and Esaulova, 2006).

In the current study we use and develop asymptotic methodology employed for the univariate case for analyzing the behavior of failure rates in the competing risk setting with a bivariate frailty.

Section 2 is devoted to basic definitions and some supplementary simple nonasymptotic properties of mixture failure rates with independent frailties.

In Section 3 we obtain explicit asymptotic results, which above, all show, that even in the case of correlated frailty the components remaining lifetimes can be considered as 'asymptotically independent' in the defined sense. In Section 4 we provide some relevant examples and discuss restrictions of our assumptions. It is worth noting that the generalization of our results to the multivariate case when $n>2$ is rather straightforward.

\section{Bivariate frailty and competing risks}

Assume that risks are dependent only via the bivariate frailty $\left(Z_{1}, Z_{2}\right)$. To construct the corresponding competing risks model consider firstly a system of two statistically independent components in series with lifetimes $T_{1} \geq 0$ and $T_{2} \geq 0$. The Cdf function of this system is

$$
F_{s}(t)=1-\bar{F}_{1}(t) \bar{F}_{2}(t)
$$

where $F_{1}(t)$ and $F_{2}(t)$ are the Cdfs of the lifetime random variables $T_{1}$ and $T_{2}$ respectively, and the survival functions $\bar{F}_{i}(t) \equiv 1-F_{i}(t)$.

Assume now that $F_{i}(t), i=1,2$ are indexed by random variables $Z_{i}$ in the following conventional sense:

$$
P\left(T_{i} \leq t \mid Z_{i}=z\right) \equiv P\left(T_{i} \leq t \mid z\right)=F_{i}(t, z), \quad i=1,2
$$

and that the pdfs $f_{i}(t, z)$ exist. Then the corresponding failure rates $\lambda_{i}(t, z)$ are $f_{i}(t, z) / \bar{F}_{i}(t, z)$.

Let $Z_{i}, i=1,2$ be interpreted as non-negative random variables with supports in $\left[a_{i}, b_{i}\right], a_{1} \geq 0, b_{i} \leq \infty$ and the pdf $\pi_{i}(z)$. 
A mixture Cdf for the $i$ th component is defined by

$$
F_{m, i}(t)=\int_{a_{i}}^{b_{i}} F_{i}(t, z) \pi_{i}(z) d z, \quad i=1,2 .
$$

The corresponding mixture failure rate is:

$$
\lambda_{m, i}(t)=\frac{\int_{a_{i}}^{b_{i}} f_{i}(t, z) \pi_{i}(z) d z}{\int_{a_{i}}^{b_{i}} \bar{F}_{i}(t, z) \pi_{i}(z) d z}=\int_{a_{i}}^{b_{i}} \lambda_{i}(t, z) \pi(z \mid t) d z,
$$

where the conditional pdf (on condition that $T_{i}>t$ ):

$$
\pi_{i}(z \mid t)=\pi_{i}(z) \frac{\bar{F}_{i}(t, z)}{\int_{a_{i}}^{b_{i}} \bar{F}_{i}(t, z) \pi_{i}(z) d z} .
$$

Assume that the components of our system are conditionally independent given $Z_{1}=z_{1}, Z_{2}=z_{2}$. Then the Cdf of the system is:

$$
F_{s}\left(t, z_{1}, z_{2}\right)=1-\bar{F}_{1}\left(t, z_{1}\right) \bar{F}_{2}\left(t, z_{2}\right)
$$

and the corresponding probability density function is

$$
f_{s}\left(t, z_{1}, z_{2}\right)=f_{1}\left(t, z_{1}\right) \bar{F}_{2}\left(t, z_{2}\right)+f_{2}\left(t, z_{2}\right) \bar{F}_{1}\left(t, z_{1}\right) .
$$

The mixture failure rate of the system in this case is defined as

$$
\begin{aligned}
\lambda_{m, s}(t) & =\frac{\int_{a_{2}}^{b_{2}} \int_{a_{1}}^{b_{1}} f_{s}\left(t, z_{1}, z_{2}\right) \pi\left(z_{1}, z_{2}\right) d z_{1} d z_{2}}{\int_{a_{2}}^{b_{2}} \int_{a_{1}}^{b_{1}} \bar{F}_{s}\left(t, z_{1}, z_{2}\right) \pi\left(z_{1}, z_{2}\right) d z_{1} d z_{2}} \\
& =\int_{a_{2}}^{b_{2}} \int_{a_{1}}^{b_{1}} \lambda_{s}\left(t, z_{1}, z_{2}\right) \pi\left(z_{1}, z_{2} \mid t\right) d z_{1} d z_{2}
\end{aligned}
$$

where

$$
\pi\left(z_{1}, z_{2} \mid t\right)=\pi\left(z_{1}, z_{2}\right) \frac{\bar{F}_{s}\left(t, z_{1}, z_{2}\right)}{\int_{a_{2}}^{b_{2}} \int_{a_{1}}^{b_{1}} \bar{F}_{s}\left(t, z_{1}, z_{2}\right) \pi\left(z_{1}, z_{2}\right) d z_{1} d z_{2}}
$$

and $\pi\left(z_{1}, z_{2}\right)$ is the bivariate joint probability density function of $Z_{1}$ and $Z_{2}$. It is clear that for our series system, defined by (8):

$$
\lambda_{s}\left(t, z_{1}, z_{2}\right)=\lambda_{1}\left(t, z_{1}\right)+\lambda_{2}\left(t, z_{2}\right) .
$$


It is clear also that if $Z_{1}$ and $Z_{2}$ are independent, which means

$$
\pi\left(z_{1}, z_{2}\right)=\pi_{1}\left(z_{1}\right) \pi_{2}\left(z_{2}\right)
$$

for some densities $\pi_{1}\left(z_{1}\right)$ and $\pi_{2}\left(z_{2}\right)$; then

$$
\pi\left(z_{1}, z_{2} \mid t\right)=\pi_{1}\left(z_{1} \mid t\right) \pi_{2}\left(z_{2} \mid t\right)
$$

which can be easily seen using definitions (7) and (11):

$$
\begin{aligned}
\pi\left(z_{1}, z_{2} \mid t\right) & =\pi_{1}\left(z_{1}\right) \pi_{2}\left(z_{2}\right) \frac{\bar{F}_{1}\left(t, z_{1}\right) \bar{F}_{2}\left(t, z_{2}\right)}{\int_{a_{2}}^{b_{2}} \int_{a_{1}}^{b_{1}} \bar{F}_{1}\left(t, z_{1}\right) \bar{F}_{2}\left(t, z_{2}\right) \pi_{1}\left(z_{1}\right) \pi_{2}\left(z_{2}\right) d z_{1} d z_{2}} \\
& =\frac{\pi_{1}\left(z_{1}\right) \bar{F}_{1}\left(t, z_{1}\right) \cdot \pi_{2}\left(z_{2}\right) \bar{F}_{2}\left(t, z_{2}\right)}{\int_{a_{1}}^{b_{1}} \bar{F}_{1}\left(t, z_{1}\right) \pi_{1}\left(z_{1}\right) d z_{1} \cdot \int_{a_{2}}^{b_{2}} \bar{F}_{2}\left(t, z_{2}\right) \pi_{2}\left(z_{2}\right) d z_{2}} \\
& =\pi_{1}\left(z_{1} \mid t\right) \pi_{2}\left(z_{2} \mid t\right) .
\end{aligned}
$$

Using equations (10) and (12)

$$
\begin{aligned}
\lambda_{m, s} & =\int_{a_{2}}^{b_{2}} \int_{a_{1}}^{b_{1}} \lambda_{s}\left(t, z_{1}, z_{2}\right) \pi\left(z_{1}, z_{2} \mid t\right) d z_{1} d z_{2} \\
& =\int_{a_{2}}^{b_{2}} \int_{a_{1}}^{b_{1}}\left[\lambda_{1}\left(t, z_{1}\right)+\lambda_{2}\left(t, z_{2}\right)\right] \pi_{1}\left(z_{1} \mid t\right) \pi_{2}\left(z_{2} \mid t\right) d z_{1} d z_{2} \\
& =\int_{a_{1}}^{b_{1}} \lambda_{1}\left(t, z_{1}\right) \pi_{1}\left(z_{1} \mid t\right) d z_{1}+\int_{a_{2}}^{b_{2}} \lambda_{2}\left(t, z_{2}\right) \pi_{2}\left(z_{2} \mid t\right) d z_{2} \\
& =\lambda_{m, 1}(t)+\lambda_{m, 2}(t)
\end{aligned}
$$

Hence, when components of the system are conditionally independent and $Z_{1}$ and $Z_{2}$ are independent, the mixture failure rate of the system is the sum of mixture failure rates of individual components.

It is worth noting that equation (13) does not hold for the case of shared frailty, when $Z_{1} \equiv Z_{2} \equiv Z$, as

$$
\begin{aligned}
\lambda_{m s} & =\frac{\int_{a}^{b} f_{s}(t, z) \pi(z) d z}{\int_{a}^{b} \bar{F}_{s}(t, z) \pi(z) d z} \\
& =\frac{\int_{a}^{b} f_{1}(t, z) \bar{F}_{2}(t, z) \pi(z) d z}{\int_{a}^{b} \bar{F}_{s}(t, z) \pi(z) d z}+\frac{\int_{a}^{b} f_{2}(t, z) \bar{F}_{1}(t, z) \pi(z) d z}{\int_{a}^{b} \bar{F}_{s}(t, z) \pi(z) d z} \\
& =\frac{\int_{a}^{b} \lambda_{1}(t, z) \bar{F}_{s}(t, z) \pi(z) d z}{\int_{a}^{b} \bar{F}_{s}(t, z) \pi(z) d z}+\frac{\int_{a}^{b} \lambda_{2}(t, z) \bar{F}_{s}(t, z) \pi(z) d z}{\int_{a}^{b} \bar{F}_{s}(t, z) \pi(z) d z}
\end{aligned}
$$


is not equal to $\lambda_{1 m}(t)+\lambda_{2 m}(t)$.

In the next section we shall study the asymptotic behavior of mixture failure rates for the most interesting case of correlated frailty.

\section{The main result}

Assume that lifetimes of both components belong to the class defined by relation (1). Let for simplicity the non-important additive term be equal to zero. The corresponding survival functions for the components are

$$
\bar{F}_{i}\left(t, z_{i}\right)=e^{-A_{i}\left(z_{i} \phi_{i}(t)\right)}, \quad i=1,2 .
$$

The following result takes place:

Theorem. Consider the competitive risks model (8). Let the corresponding survival functions be defined by equation (14).

Suppose that the mixing variables $Z_{1}$ and $Z_{2}$ have a joint probability density function $\pi\left(z_{1}, z_{2}\right)$, which is defined in $\left[0, b_{1}\right] \times\left[0, b_{2}\right], 0<b_{1}, b_{2} \leq \infty$.

Let the following properties hold:

(a) $\pi\left(z_{1}, z_{2}\right)=z_{1}^{\alpha_{1}} z_{2}^{\alpha_{2}} \pi_{0}\left(z_{1}, z_{2}\right)$, where $\alpha_{1}, \alpha_{2}>-1$.

(b) $\pi_{0}\left(z_{1}, z_{2}\right)$ is continuous at $(0,0), \pi_{0}(0,0) \neq 0$.

(c) $A_{i}(s), i=1,2$ are positive ultimately increasing differentiable functions,

$$
\int_{0}^{\infty} e^{-A_{i}(s)} s^{\alpha_{i}} d s<\infty
$$

Assume finally that $\phi_{1}(t), \phi_{2}(t) \rightarrow \infty$ as $t \rightarrow \infty$.

Then

$$
\lambda_{m, s}(t) \sim\left(\alpha_{1}+1\right) \frac{\phi_{1}^{\prime}(t)}{\phi_{1}(t)}+\left(\alpha_{2}+1\right) \frac{\phi_{2}^{\prime}(t)}{\phi_{2}(t)} .
$$

By the sign $\sim$ we, as usually, denote the asymptotic equivalence: $g_{1}(t) \sim$ $g_{2}(t)$ as $t \rightarrow \infty$ means $g_{1}(t) / g_{2}(t) \rightarrow 1$ as $t \rightarrow \infty$.

Remark. It follows from relation (13) and the corresponding result in the univariate case (Finkelstein and Esaulova, 2006) that for the univariate mixing densities

$$
\tilde{\pi}_{i}(z)=z^{\alpha_{i}} \tilde{\pi}_{i, 0}(z), \quad \alpha_{i}>-1
$$


where $\tilde{\pi}_{i, 0}(z)$ are bounded in $[0, \infty)$, continuous and positive at $z=0$, the mixture failure rate of our system with independent frailties is given by the following asymptotic formula:

$$
\lambda_{m, s}(t)=\lambda_{m, 1}(t)+\lambda_{m, 2}(t) \sim \tilde{\lambda}_{m, 1}(t)+\tilde{\lambda}_{m, 2}(t),
$$

where $\tilde{\lambda}_{m, i}(t)$ is a mixture failure rate of a family with survival functions given by (14) with the mixing density $\tilde{\pi}_{i}(z), i=1,2$.

Comparing this relation with the result of the Theorem, it is easy to arrive at the following important interpretation:

Under certain assumptions the asymptotic mixture failure rate in the correlated frailty model with conditionally independent components is equivalent to the asymptotic mixture failure rate in the independent frailty model.

This can be also viewed as some asymptotic independence of remaining lifetimes of our components in the correlated frailty model.

Proof. We start our proof with the following supplementary lemma:

Lemma. Let $g\left(z_{1}, z_{2}\right)$ be a nonnegative integrable function in $[0, \infty)^{2}$. Let $h\left(z_{1}, z_{2}\right)$ be a nonnegative locally integrable function defined in $[0, \infty)^{2}$, such that it is bounded everywhere and continuous at the origin.

Then, as $t_{1} \rightarrow \infty, t_{2} \rightarrow \infty:$

$$
t_{1} t_{2} \int_{0}^{\infty} \int_{0}^{\infty} g\left(t_{1} z_{1}, t_{2} z_{2}\right) h\left(z_{1}, z_{2}\right) d z_{1} d z_{2} \rightarrow h(0,0) \int_{0}^{\infty} \int_{0}^{\infty} g\left(z_{1}, z_{2}\right) d z_{1} d z_{2} .
$$

Proof. The proof is rather straightforward:

$$
\begin{aligned}
t_{1} t_{2} \int_{0}^{\infty} \int_{0}^{\infty} g\left(t_{1} z_{1},\right. & \left.t_{2} z_{2}\right) h\left(z_{1}, z_{2}\right) d z_{1} d z_{2} \\
= & \int_{0}^{\infty} \int_{0}^{\infty} g\left(z_{1}, z_{2}\right) h\left(\frac{z_{1}}{t_{1}}, \frac{z_{2}}{t_{2}}\right) d z_{1} d z_{2} .
\end{aligned}
$$

Indeed, $h\left(z_{1}, z_{2}\right)$ is bounded; assume that it is bounded by some $M$. The function $g\left(z_{1}, z_{2}\right)$ is integrable, then for any $\epsilon>0$ there is a finite $b>0$, such that

$$
\iint_{[0, \infty)^{2}-[0, b]^{2}} g\left(z_{1}, z_{2}\right) d z_{1} d z_{2}<\epsilon
$$


Then

$$
\begin{gathered}
\left|\int_{0}^{\infty} \int_{0}^{\infty} g\left(z_{1}, z_{2}\right)\left[h\left(\frac{z_{1}}{t_{1}}, \frac{z_{2}}{t_{2}}\right)-h(0,0)\right] d z_{1} d z_{2}\right| \\
\leq \int_{0}^{b} \int_{0}^{b} g\left(z_{1}, z_{2}\right)\left|h\left(\frac{z_{1}}{t_{1}}, \frac{z_{2}}{t_{2}}\right)-h(0,0)\right| d z_{1} d z_{2} \\
+2 M \iint_{[0, \infty)^{2}-[0, b]^{2}} g\left(z_{1}, z_{2}\right) d z_{1} d z_{2} .
\end{gathered}
$$

The first double integral tends to zero since $h\left(z_{1}, z_{2}\right)$ is continuous at $(0,0)$, and the second can be made arbitrarily small.

Now we can proceed with the proof itself. Substituting (8) and (9) into (10) we get

$$
\begin{aligned}
\lambda_{m, s}(t)= & \frac{\int_{0}^{b_{1}} \int_{0}^{b_{2}} f_{1}\left(t, z_{1}\right) \bar{F}_{2}\left(t, z_{2}\right) \pi\left(z_{1}, z_{2}\right) d z_{2} d z_{1}}{\int_{0}^{b_{1}} \int_{0}^{b_{2}} \bar{F}_{1}\left(t, z_{1}\right) \bar{F}_{2}\left(t, z_{2}\right) \pi\left(z_{1}, z_{2}\right) d z_{2} d z_{1}} \\
& +\frac{\int_{0}^{b_{2}} \int_{0}^{b_{1}} f_{2}\left(t, z_{2}\right) \bar{F}_{1}\left(t, z_{1}\right) \pi\left(z_{1}, z_{2}\right) d z_{1} d z_{2}}{\int_{0}^{b_{2}} \int_{0}^{b_{1}} \bar{F}_{2}\left(t, z_{1}\right) \bar{F}_{1}\left(t, z_{1}\right) \pi\left(z_{1}, z_{2}\right)}
\end{aligned}
$$

Denote the first term on the right-hand side by $\lambda_{m, s}^{1}(t)$ and the second one by $\lambda_{m, s}^{2}(t)$. Then

$$
\lambda_{m, s}(t)=\lambda_{m, s}^{1}(t)+\lambda_{m, s}^{2}(t) .
$$

Consider $\lambda_{m, s}^{1}(t)$ and $\lambda_{m, s}^{2}(t)$ separately. The probability density function of $T_{1}$ is

$$
f_{1}\left(t, z_{1}\right)=A_{1}^{\prime}\left(z_{1} \phi_{1}(t)\right) z_{1} \phi_{1}^{\prime}(t) e^{-A_{1}\left(z_{1} \phi_{1}(t)\right)}
$$

and

$$
\lambda_{m, s}^{1}(t)=\frac{\int_{0}^{b_{1}} \int_{0}^{b_{2}} A_{1}^{\prime}\left(z_{1} \phi_{1}(t)\right) z_{1} \phi_{1}^{\prime}(t) e^{-A_{1}\left(z_{1} \phi_{1}(t)\right)-A_{2}\left(z_{2} \phi_{2}(t)\right)} \pi\left(z_{1}, z_{2}\right) d z_{2} d z_{1}}{\int_{0}^{b_{1}} \int_{0}^{b_{2}} e^{-A_{1}\left(z_{1} \phi_{1}(t)\right)-A_{2}\left(z_{2} \phi_{2}(t)\right)} \pi\left(z_{1}, z_{2}\right) d z_{2} d z_{1}}
$$

Applying the Lemma to the numerator, we see that it is asymptotically equivalent to

$$
\frac{\phi_{1}^{\prime}(t) \pi_{0}(0,0)}{\phi_{1}(t)^{\alpha_{1}+2} \phi_{2}(t)^{\alpha_{2}+1}} \int_{0}^{\infty} A_{1}^{\prime}(u) u^{\alpha_{1}+1} e^{-A_{1}(u)} d u \int_{0}^{\infty} s^{\alpha_{2}} e^{-A_{2}(s)} d s
$$


and the denominator is equivalent to

$$
\frac{\pi_{0}(0,0)}{\phi_{1}(t)^{\alpha_{1}+1} \phi_{2}(t)^{\alpha_{2}+1}} \int_{0}^{\infty} u^{\alpha_{1}} e^{-A_{1}(u)} d u \int_{0}^{\infty} s^{\alpha_{2}} e^{-A_{2}(s)} d s
$$

Hence,

$$
\lambda_{m, s}^{1}(t) \sim \frac{\phi_{1}^{\prime}(t)}{\phi_{1}(t)} \cdot \frac{\int_{0}^{\infty} A_{1}^{\prime}(u) u^{\alpha_{1}+1} e^{-A_{1}(u)} d u}{\int_{0}^{\infty} u^{\alpha_{1}} e^{-A_{1}(u)} d u}
$$

Due to condition (c) of the Theorem

$$
e^{-A(s)} s^{\alpha+1} \rightarrow 0 \quad \text { as } s \rightarrow \infty .
$$

Indeed, by the mean value theorem:

$$
\int_{s}^{2 s} e^{-A(u)} u^{\alpha} d u=s e^{-A\left(s_{1}\right)} s_{1}^{\alpha}
$$

for some $s \leq s_{1} \leq 2 s$. The right-hand side tends to 0 . For $s$ larger than some $s_{0}$ we have $A\left(s_{1}\right)>A(s)$; thus, the left-hand side is smaller than $2^{\alpha} s^{\alpha+1} e^{-A(s)}$, which leads to (20). Using it while integrating by parts, we get

$$
\int_{0}^{\infty} A^{\prime}(s) e^{-A(s)} s^{\alpha+1} d s=(\alpha+1) \int_{0}^{\infty} e^{-A(s)} s^{\alpha} d s .
$$

Thus, from (19)

$$
\lambda_{m, s}^{1}(t) \sim\left(\alpha_{1}+1\right) \frac{\phi_{1}^{\prime}(t)}{\phi_{1}(t)}
$$

Similarly,

$$
\lambda_{m, s}^{2}(t) \sim\left(\alpha_{2}+1\right) \frac{\phi_{2}^{\prime}(t)}{\phi_{2}(t)}
$$

\section{Some examples}

Assumptions (a) and (b) of the Theorem impose certain restrictions on the mixing distribution. In the univariate case the corresponding conditions (16) are satisfied for a wide class of distributions, such as Gamma, Weibull, etc. (Finkelstein and Esaulova, 2006). In the bivariate case they obviously hold for all densities that are positive and continuous at the origin. 
We will present now a couple of simple examples. But first, we will interpret our results in terms of copulas, which can be helpful in analyzing the competing risks problems.

The following result is obvious and therefore its proof is omitted:

Proposition. Assume that the bivariate mixing Cdf is given by the copula $C(u, v)$ :

$$
\Pi\left(z_{1}, z_{2}\right)=C\left(\Pi_{1}\left(z_{1}\right), \Pi_{2}\left(z_{2}\right)\right),
$$

where $\Pi_{1}\left(z_{1}\right), \Pi_{2}\left(z_{2}\right)$ are univariate $C d f$ s, satisfying similar univariate conditions (see (16)) with nonnegative power indices $\alpha_{1}, \alpha_{2} \geq 0$. Then the bivariate conditions are satisfied, if $c(u, v)=\frac{\partial^{2} C}{\partial u \partial v}(u, v)$ can be represented as

$$
c(u, v)=u^{\gamma_{1}} v^{\gamma_{2}} c_{0}(u, v),
$$

where $c_{0}(u, v)$ is continuous and positive at $(0,0), \gamma_{1}, \gamma_{2} \geq 0$.

\section{Example 1. Farlie-Gumbel-Morgenstern copula.}

The distribution is defined via the copula

$$
C(u, v)=u v(1+\theta(1-u)(1-v))
$$

where $|\theta| \leq 1, u, v \in[0,1]$. Since

$$
\frac{\partial^{2} C}{\partial u \partial v}(u, v)=1+\theta(1-2 u)(1-2 v)
$$

is continuous at the origin and positive there if $\theta>-1$, the bivariate conditions hold when $-1<\theta \leq 1$. Therefore, the results of the Theorem hold if the univariate Cdfs belong to the admissible class.

The next example considers a bivariate distribution defined directly.

Example 2. Gumbel's bivariate exponential distribution.

The joint cumulative distribution function is defined by

$$
\Pi\left(z_{1}, z_{2}\right)=1-e^{-z_{1}}-e^{-z_{2}}+e^{-z_{1}+z_{2}+\theta z_{1} z_{2}}
$$

and the joint density function by

$$
\pi\left(z_{1}, z_{2}\right)=e^{-z_{1}+z_{2}+\theta z_{1} z_{2}}\left\{\left(1+\theta z_{1}\right)\left(1+\theta z_{2}\right)-\theta\right\},
$$

where $0 \leq \theta \leq 1, z_{1}, z_{2}>0$. 
The pdf is obviously bounded in $[0, \infty)^{2}$ and continuous everywhere, $\pi(0,0)=1-\theta$. Thus for $\theta<1$ the mixing pdf satisfies the considered conditions and asymptotic relation (15) holds.

Other distributions that meet the conditions are Dirichlet distribution (Kotz et al, p. 485) and inverted Dirichlet distribution (Kotz et al, p. 491), some types of multivariate logistic distributions (Kotz et al, p. 551), some types of special bivariate extreme value distributions (Kotz et al, p. 625).

There are also examples, in which the conditions of the Theorem do not hold. This happens, e.g., when the joint Cdf depends on $\max \left(z_{1}, z_{2}\right)$ and is not absolutely continuous. The widely used Marshall and Olkin's bivariate exponential with the survival function

$$
\bar{\Pi}\left(z_{1}, z_{2}\right)=e^{-\gamma_{1} z_{1}-\gamma_{2} z_{2}-\gamma_{12} \max \left(z_{1}, z_{2}\right)}
$$

is a relevant example. Some multivariate Weibull distributions also employ $\max$ functions and are not absolutely continuous at $(0,0)$. The corresponding examples can be found in Kotz, Balakrishnan, and Johnson (2000) (p. 431).

Finally, in order to illustrate explicitly the main result of this paper given by by the Theorem, assume that the lifetimes of both components can be described by the PH model (2) and $\alpha_{1}=\alpha_{2}=0$. Then, as $t \rightarrow \infty$, in accordance with (15)

$$
\lambda_{m, s}(t) \sim \frac{\lambda_{1}(t)}{\int_{0}^{t} \lambda_{1}(u) d u}+\frac{\lambda_{2}(t)}{\int_{0}^{t} \lambda_{2}(u) d u},
$$

which is speaking for itself simple asymptotic formula.

If the lifetimes of both components are described by the ALM model (3), then the asymptotics is surprisingly simple:

$$
\lambda_{m, s}(t) \sim \frac{2}{t}
$$

Both of these formulas show that in this case $\left(\alpha_{1}=\alpha_{2}=0\right)$ asymptotic behavior does not depend on the mixing distribution at all.

\section{References}

[1] Badia, F.G., Berrade, M.D., Campos C.A., and Navascues, M.A. (2001). On the behavior of aging characteristics in mixed populations, Probability in the Engineering and Informational Sciences, 15, 83-94. 
[2] Block, H. W., Mi, J., and Savits, T. H. (1993). Burn-in and mixed populations. Journal of Applied Probability, 30, 692-702.

[3] Block, H.W., Savits, T.N. and Wondmagegnehu, E. T. (2003). Mixtures of distributions with increasing linear failure rates. Journal of Applied Probability, 40, 485-504.

[4] Finkelstein, M.S., and Esaulova, V (2001), Modeling a failure rate for the mixture of distribution functions Probability in Engineering and Informational Sciences, 15, 383-400.

[5] Finkelstein, M.S., and Esaulova, V (2006), Asymptotic behavior of a general class of mixture failure rates, the Advances in Applied Probability, 38, 1, 244-262.

[6] S. Kotz, N. Balakrishnan, N. L. Johnson. Continuous Multivariate Distributions, Models and Applications. Vol. 1, 2000. Wiley, New York.

[7] Lynch, J.D (1999). On conditions for mixtures of increasing failure rate distributions to have an increasing failure rate. Probability in the Engineering and Informational Sciences, 13, 33-36.

[8] Shaked, M and Spizzichino, F. (2001). Mixtures and monotonicity of failure rate functions. In: Advances in Reliability (N. Balakrishnan and C.R. Rao -eds.),Vol. 20, Elsevier: Amsterdam. 185-198 